\title{
Rapid neurological deterioration in a 22-year-old man
}

\author{
MO McCarron, GV McDonnell, JM Gibson
}

A previously healthy 22-year-old right-handed man presented with a four month history of stumbling to the left side. His parents had also noticed hand clumsiness, difficulty dressing and impaired vision. Speech and memory had deteriorated. History was unremarkable apart from measles at the age of five years.

On examination, speech was slow and lacking spontaneity but he was orientated and not dysphasic. Visual acuity was $6 / 9$ bilaterally. There was a left homonymous hemianopia, pseudoathetosis of the left hand and left-sided ataxia. Investigations included a normal computed tomography (CT) scan of brain six weeks previously. Magnetic resonance imaging (MRI) of brain on admission is shown below (figure 1). Electroencephalogram (EEG) revealed periodic bursts of high-amplitude sharp and slow wave activity at $2 \mathrm{~Hz}$, lasting one second and repeating every 10 to 15 seconds.

The patient became bed-bound within one week with increasing left-sided weakness and spasticity. By day 14 there was cortical blindness, marked left hemiplegia and leftward gaze palsy. Stabilisation briefly ensued, followed by further deterioration. Rhythmic myoclonic jerks every 10 seconds developed in the head, neck and right side of the body. MRI on day 45 is shown in figure 2 . Subsequently he has remained blind, mute and bed-bound requiring total care.

Institute of Neurological Sciences, Southern General Hospital, Glasgow, UK MO McCarron GV McDonnell

Department of Neurology, Royal Victoria Hospital, Belfast, N Ireland JM Gibson

Correspondence to Dr JM Gibson, Department of Neurology, Ward 21, Quin House, Royal Victoria Hospital, Grosvenor Road, Belfast BT12 6BA, N Ireland

Accepted 27 June 1996

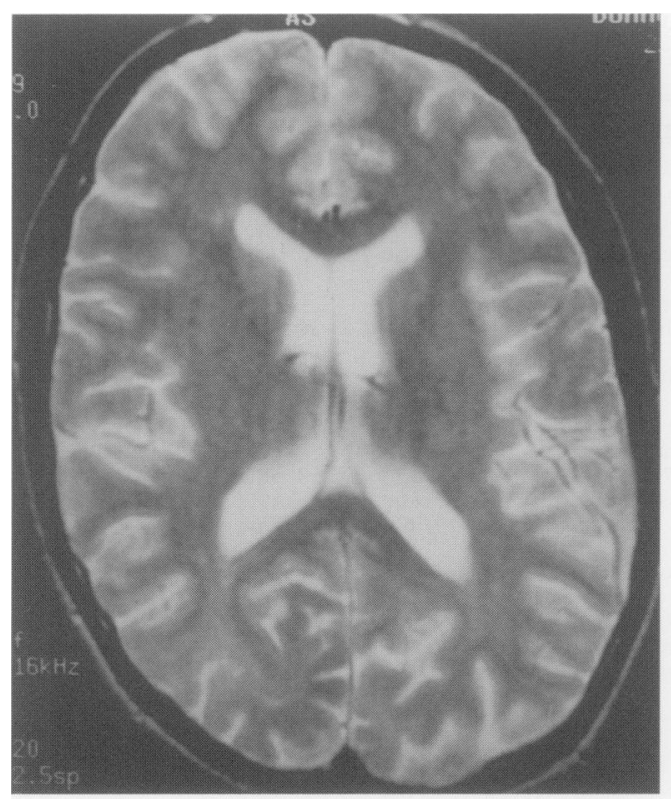

A

Figure 1 Axial T2-weighted MRI (TR $400 \mathrm{~ms} / \mathrm{TE} 81 \mathrm{~ms}$ ) performed on admission 


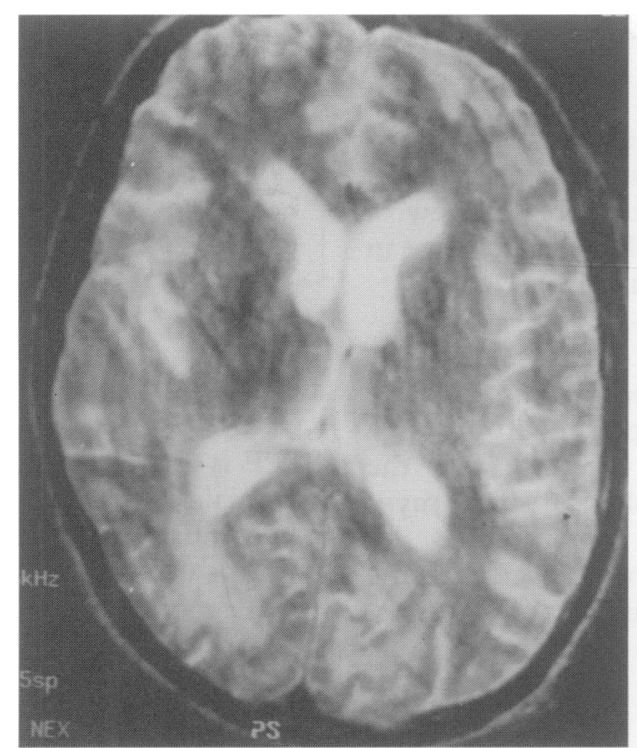

A

Figure 2 Repeat MRI scan six weeks later

\section{Questions}

1 What do the second MRI scans (figure 2) show? 2 What is the diagnosis?

3 What further investigation would you perform?

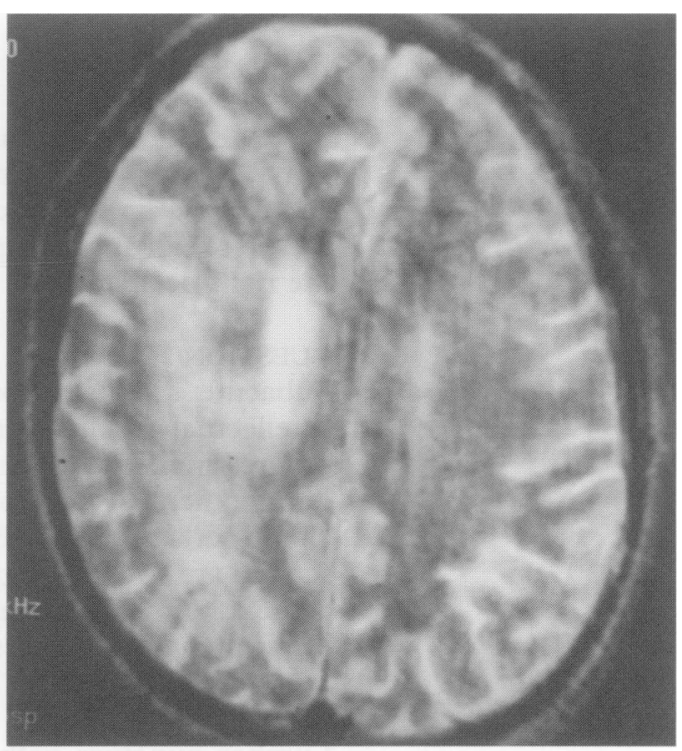

B 
Answers

QUESTION 1

The MRI in figure 2 shows extensive increased signal in the white matter of the right cerebral hemisphere and left occipital lobe. Review of the first scan suggests possible early change in the right occipital lobe (figure 1A).

\section{QUESTION 2}

Subacute sclerosing panencephalitis (SSPE). Intellectual decline and personality change progressing to dementia, together with the development of focal neurological signs, myoclonic jerks and the EEG abnormalities described are strongly suggestive, especially in the context of a previous history of measles. Typical clinical features are listed in box 1 .

\section{QUESTION 3}

Lumbar puncture with measurement of cerebrospinal fluid (CSF) and serum measles antibody titres. The diagnosis of SSPE can be made solely on the basis of this investigation and the clinical findings. ${ }^{1} \mathrm{CSF}$ and serum measles antibody titres were $1 / 200$ and $1 / 1600$, ratio $1: 8$, confirming the diagnosis of SSPE. The full diagnostic criteria are given in box 1 .

\section{Treatment}

From day 11 interferon alpha-2b was administered intraventricularly at 1 million units (MU) daily for one week, then $3 \mathrm{MU}$ weekly. Isoprinosine, $100 \mathrm{mg} / \mathrm{kg} /$ day, was given orally. Because of the continuing deterioration, subsequent development of pyrexia and their unproven efficacy in adults, both drugs were discontinued on day 44 .

\begin{tabular}{|l|}
\hline Subacute sclerosing panencephalitis \\
\hline Clinical features \\
- intellectual deterioration \\
- personality/behavioural changes \\
- speech disturbance \\
- myoxia \\
- generalised/focal seizures \\
- visual loss \\
Diagnostic criteria \\
- clinical findings \\
anti-measles antibody and oligoclonal \\
- Eanding in CSF \\
- histology: intranuclear inclusion bodies or \\
measles antigen \\
Differential diagnosis \\
- progressive multifocal leukoencephalopathy: \\
- C virus in immunodeficiency eg, AIDS \\
- Creutzfeldt-Jakob disease \\
- Schilder's disease \\
- metabolic encephalopathy \\
- acute disseminated encephalomyelitis \\
- vafora-body disease: adolescent seizures \\
- viral encephalitis \\
\hline
\end{tabular}

Box 1

\section{Learning points}

- mental slowing may be the first symptom of SSPE

- ask about measles and measles vaccine if diagnosis considered

- initial neuroimaging may be normal

Box 2

\section{Discussion}

SSPE is characterised by an insidious onset of personality change, motor dysfunction and myoclonus. An inexorable downhill course is typical, although spontaneous remission has been reported. The age of onset of SSPE ranges from 2 to 27 years (median 10.5 years) and the risk in England and Wales between 1970 and 1989 was estimated to be 4 per 100000 measles cases, the risk being greatest if measles occurs under one year of age. ${ }^{2}$

Vaccination against measles began in 1968, but uptake was low, especially in rural areas such as Wales ${ }^{3}$ and Northern Ireland. The MMR vaccination at 15 months has had a much higher uptake, approaching $80 \%$, resulting in fewer measles notifications. In 1994 an intensive measles and rubella vaccination campaign targeted over 7 million schoolchildren aged between five and 16 years in England which averted an anticipated measles epidemic. ${ }^{4}$ The Chief Medical Officer encouraged reporting of adverse reactions, but serious suspected cases were very rare. One case of SSPE developed a month after immunisation. However, this was probably due to wild measles because a measles history existed and SSPE usually has a much longer incubation period. Farrington estimated an almost 30 -fold reduction in SSPE with vaccination, ${ }^{5}$ the remaining risk attributable to previous subclinical measles or vaccine failure.

The treatment of SSPE is controversial. Immunomodulators including cimetidine, isoprinosine and alpha interferon have all been used. Decreased levels of endogenous interferon in CSF and sera in SSPE patients explain the rationale for its use. In one study, eight out of 18 children (44\%) remained stable or improved on combined oral isoprinosine and intraventricular alpha interferon. ${ }^{6}$ Only three definitely improved and these had a slowly progressive onset (unlike our patient). It is recognised that SSPE has a more rapid progression and worse prognosis in adults. ${ }^{7}$ Adverse effects of intraventricular interferon include febrile reactions, lethargy, ventriculitis/ meningitis and stroke.

Neuroimaging is not always helpful, especially in the early stages. Chronological single photon emission CT studies of a patient with SSPE have shown abnormalities before MRI and CT scanning. ${ }^{8}$ MRI scans typically show white matter changes around the occipital and frontal horns. Progression of disease extends these white matter changes periventricularly. Improvement has been seen with intrathecal alpha-interferon. ${ }^{7}$ Ventricular dilation and gyral atrophy occur later. 
In conclusion, SSPE is a rare progressive brain disease due to a mutated measles virus. The diagnosis is easily overlooked because of an insidious or bizarre presentation together with normal neuroimaging. The variable natural history, where spontaneous remissions may occur, creates major problems for small therapeutic trials. However, given its catastrophic course in the young, adequate evaluation of the various treatment options in a controlled prospective international study is long overdue.

1 Bellman MH, Dick G. Surveillance of subacute sclerosing panencephalitis. $B M \mathcal{F} 1980$; ii: $393-4$.

2 Millar C, Farrington CP, Harbert K. The epidemiology of SSPE in England and Wales 1970-1989. Int $\mathcal{F}$ Epidemiol 1992; 21: $998-1006$.

3 Sussman J, Compston DAS. Subacute sclerosing panencephalitis in Wales. $Q 7 \mathrm{Med}$ 1994; 87: 23-34.

4 Cuffs F. Revaccination against measles and rubella. $B M \mathcal{F}$ 1996; 312: $589-90$.

5 Farrington CP. Subacute sclerosing panencephalitis in England and Wales: transient effects and risk estimates. Stat Med 1991; 10: $1733-44$

\section{Final diagnosis}

Subacute sclerosing panencephalitis.

Keywords: subacute sclerosing panencephalitis, interferon-alpha, isoprinosine

We are grateful to Dr CS McKinstry, Consultant Neuroradiologist.
6 Gascon G, Yamani S, Cromwell J, et al. Combined oral isoprinosine-intraventricular alpha-interferon therapy for SSPE. Brain Dev 1993; 15: 346-55.

7 Tan E, Namer IJ, Ciger A, Zileli T, Kucukali T. The prognosis of subacute sclerosing panencephalitis in adults. Report of 8 cases and review of the literature. Clin Neurol Neurosurg 1991; 93: 205-9.

8 Yagi S, Miura S, Mizuta S, et al. Chronological SPECT studies of a patient with subacute sclerosing panencephalitis. Brain Dev 1993; 15: $141-5$.

\title{
Mental dullness after thrombolytic therapy
}

\author{
KS Woo, HCK Chan, WS Poon
}

\section{The Chinese}

University of Hong

Kong, Prince of

Wales Hospital,

Shatin, Hong Kong

Department of

Medicine

KS Woo

HCK Chan

Department of

Surgery

WS Poon

Accepted 17 July 1996
A 68-year-old hypertensive man was admitted to the coronary care unit with acute inferior myocardial infarction presenting with sudden severe gripping central chest pain for two hours and ST elevation at inferior leads of electrocardiogram. Recombinant tissue plasminogen activator (rtPA) $100 \mathrm{mg}(10 \mathrm{mg}$ bolus, $50 \mathrm{mg}$ infusion in one hour and then $40 \mathrm{mg}$ infusion over two hours) was given intravenously, followed by intravenous heparin (4000 units bolus then 800 units/h) and isosorbide dinitrate. Morphine and oral aspirin were also given. The patient responded well. His chest pain subsided soon after admission. There was early peaking of the creatine phosphokinase 14 hours after onset of chest pain. However, he started to appear mentally dull 20 hours after receiving the rtPA. His conscious state deteriorated rapidly over the next two hours.

\section{Questions}

1 What is the most probable cause of his mental dullness?

2 What further investigation would you undertake to confirm this? 